\title{
Revista de Saúde
}

\section{Revista Semestral da Universidade Severino Sombra}

(C) Universidade Severino Sombra

Todos os direitos reservados. É permitida a reprodução parcial ou total desta obra, desde que citada a fonte e que não seja para venda ou qualquer fim comercial. Os textos publicados na Revista de Saúde são de responsabilidade de seus autores. As informações neles contidas, bem como as opiniões emitidas, não representam pontos de vista da Universidade Severino Sombra.

\author{
Presidente da Fundação Educacional Severino Sombra \\ Eng. Marco Antonio Vaz Capute \\ Reitor da Universidade Severino Sombr \\ Prof. Dr. Marco Antonio Soares de Souza \\ Pró-Reitor de Pesquisa e Pós-Graduação e Editor-Chefe \\ Prof. Dr. Carlos Eduardo Cardoso \\ Editora Executiva da Revista de Saúde \\ Prof. ${ }^{\mathrm{a}}$ Dr. ${ }^{\mathrm{a}}$ Maria Fernanda de Melo Costa
}

\section{Conselho Editorial}

Prof. Dr. Daniel Augusto Barroso Lessa (UFF)

Dr, Johnatas Dutra Silva (UFRJ)

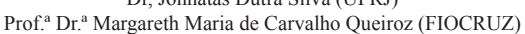

Prof. ${ }^{a}$ Dr. ${ }^{a}$ Maria Fernanda de Melo Costa (Wintec/USS)

Prof. ${ }^{\text {a }}$ Dr. Nayro Xavier de Alencar (UFF)

\section{Avaliadores $\boldsymbol{A d}$ hoc}

Alan Gomes Miranda (USS)

Alessandra Soares Nunes Tovar Elias (USS)

Ana Paula de Almeida (USS)

Carla Cristina Neves Barbosa (USS

Daniel Augusto Barroso Lessa (UFF)

Eduardo Tavares Lima Trajano (USS)

Fátima Niemeyer da Rocha (USS)

Fernanda Cabral Samico (USS)

Guilherme Marques Soares (USS)

Gerson Pereira Silva

Gustavo Mendes Gomes (USS)

João Carlos de Souza Côrtes Júnior (USS)

Johnatas Dutra Silva (UFRJ)

Lidiane de Castro Soares (USS)

Marcia Torres Ramos (UFRRJ)

Apoio, Realização, Elaboração e Edição Pró-Reitoria de Pesquisa e Pós-Graduação

Editor Responsáve

Prof. Dr. Carlos Eduardo Cardoso

Projeto Gráfico / Arte da capa

Natalia da Silveira Santos

Diagramação e Editoração Eletrônica

Maria Paula Viana Baptista
Marcelo Flores Catelli (USS) Marcelo Flores Catelli (USS)

Marcos Antonio Mendonça (USS)

Margareth Maria de Carvalho Queiroz (FIOCRUZ/USS)

Maria Fernanda de Mello Costa (USS)

Maria Cristina Almeida de Souza (USS

Marilei de Melo Tavares Souza (USS)

Marise Maleck (USS)

Melissa Manna Marques (USS)

Mônica de Almeida Carreiro (USS/UNIRIO)

Nayro Xavier de Alencar (UFF)

Sebastião Jorge da Cunha Gonçalves (USS)

Sebastião Jorge da Cunha G

Sileno Corrêa Brum (USS)

Tereza Aparecida Ferreira Dornelas (FAETERJ)

Vinicius Marins Carraro (USS)
Equipe

http://editorauss.uss.br/index.php/RS/about/displayMembership/11

Editora da Universidade Severino Sombra

Av. Expedicionário Oswaldo de Almeida Ramos, 280, Bloco 3 , $2^{\circ}$ andar - Centro, Vassouras-RJ, CEP: 27700-000

Tel.: (24) 2471-8373

E-mail: editorauss@uss.br 